\title{
Development of Lighting Control System for Smart Hotel Rooms
}

\author{
Jiajia Feng, Yongjie Yang*, Hongming Shen, Zhitian Cai \\ ${ }^{a}$ School of Electronics and Information, Nantong University, Nantong 226019, China
}

\begin{abstract}
In order to realize the scientific management of the light environment and achieve the purpose of saving energy and increasing efficiency for the hotel, this paper designs and implements an intelligent lighting control system for the room, manual control and automatic control of dual management for the room lighting [6]. The system uses the multi-layer distributed structure, combining organically with the embedded technology, Internet of things technology, sensor technology, CAN bus technology and so on, and the embedded ARM microprocessor is the core of the system, combining with the intelligent gateway module, I/O weak current digital input and output module, dimming module, illuminance sensor module and power module, and communicating with each other by the CAN bus, to realize the intelligent management of the room lighting. The Lighting Control System for Smart Hotel Rooms is facing up to the high star hotel, to improve the management level and service quality of the hotel, and save energy, scientific and intelligent management, and enhance the competitiveness for the hotel, it will be an important part of the Smart Hotel System which has a certain application value [14]. As a new and most promising light source, all kinds of lighting have attracted more and more attention for their energy saving, environmental protection and other advantages.
\end{abstract}

Keywords: lighting control; ARM microprocessor; dimming; CAN bus; intelligent management

(Submitted on July 25, 2017; Revised on August 30, 2017; Accepted on September 15, 2017)

(This paper was presented at the Third International Symposium on System and Software Reliability.)

(C) 2017 Totem Publisher, Inc. All rights reserved.

\section{Introduction}

In the Internet of things, cloud computing and other new information technology in the rapid development of the background, "Smart Hotel" era quietly coming. Smart hotel is a perfect hotel intelligent system, realize the digital hotel information service through the digital and network technology, information and intelligence, to meet the personalized needs of customers, help the hotel achieve the purpose of energy saving and efficiency [12]. The intelligent control of room lighting is an important aspect of hotel management. The realization of intelligent indoor lighting is a traditional lighting control system upgrade; people are no longer confined to ordinary light. Depending on different times of the day, there's the use of outdoor brightness or an area of the room, light automatic control. Its emergence not only creates a good living environment for people, but also improves the quality of life, meets the requirements of energy conservation and emission reduction, and makes room management more simple, efficient and orderly.

The remainder of the present study is structured as follows: in section 2, the overall structure of system on author work. In section 3, a detailed introduction is presented to explain the design methods. In section 4, the experimental setup and results, and the discussion are presented. In section 5, the conclusion is given.

\section{Overall architecture}

The lighting control system for smart hotel rooms adopts multi-layer distribution structure. Among them, the terminal layer is the core of the whole system. Each terminal sink node has manual and automatic dimming functions [2]. Its main function is to achieve:

\footnotetext{
* Corresponding author.

E-mail address: yang.yj@ntu.edu.cn.
} 
The other function modules take the STM32F407 as the main controller. The dimming module of the system is divided into the silicon controlled dimming module and 1-10V dimming module. Among them, the silicon controlled dimming module is mainly for incandescent lamps, adjusting the effective value of the voltage by chopping the sine wave of the input voltage to adjust the illuminance; $1-10 \mathrm{~V}$ dimming module is mainly for LED, filtering and D/A conversion through PWM modulation to adjust the illuminance [15].

The illuminance sensor module mainly uses the lighting digital sensor OPT3002 to detect the illumination intensity of the outside visible lighting range to adjust the illumination automatically. In particular, the illuminance sensor module is mainly induced by the external light, and the illuminance data is collected and transmitted to the dimming module through the CAN bus, so that the room lighting can be automatically adjusted.

The system takes hotel room lighting as the research object. Combined with the requirement in the hotel management, the system designs and develops a kind of Intelligent Hotel Room lighting control system, so as to realize the automatic control and manual control of the room lighting [1,11]. The system uses the ARM microprocessor as the core, with CAN bus as the communication network, connecting each module. The whole system is mainly composed of terminal control part and sampling part, so as to realize the adjustment of light. The overall structure of system is shown in Figure 1.

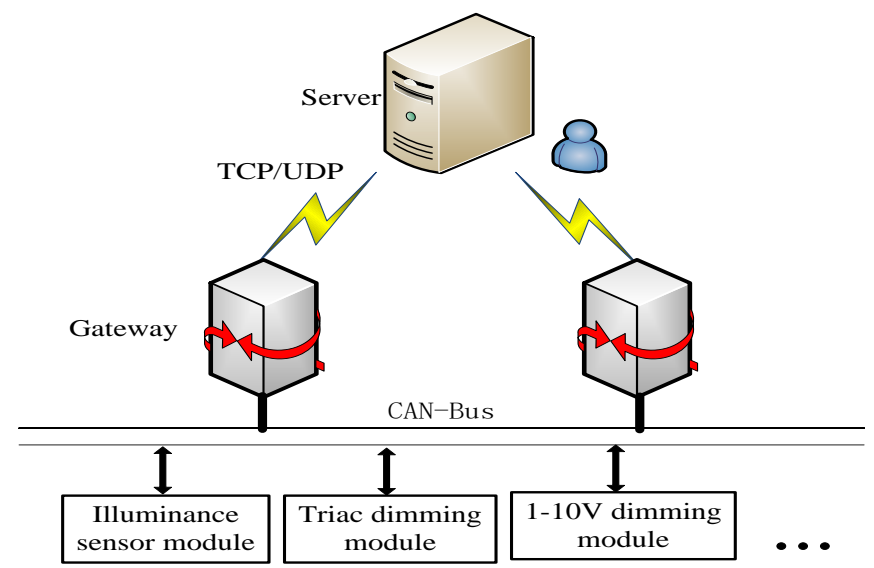

Figure 1. The overall structure of system

\section{Method}

\subsection{Hardware Design of System}

The hardware design of the system is mainly aimed at the control and acquisition part of the terminal layer, which is the basis of system function implementation. The rationality of hardware design of system determines the system performance to a great extent [10]. Here mainly is the electronic technology, the PCB drawing technology and the signal processing technology fusion.

\subsubsection{Hardware Design of Control Part}

According to the requirement of system function realization, the control terminal part mainly realizes dimming function. Its frame is shown in Figure 2. The control terminals are mainly used for adjusting the lamps and lanterns of different materials, such as incandescent lamps and LED. The core part is the 1-10 dimming circuit and the thyristor dimming circuit.

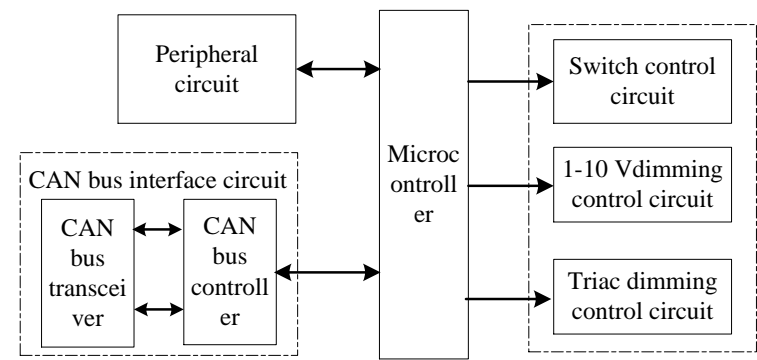

Figure 2. The hardware structure of control part 
When ordinary incandescent lamp is illuminated, the system USES silicon controlled to adjust the light. The modulation of traditional transformer and rheostat, although changing the amplitude of sinusoidal alternating current, has not really changed the nature of the sinusoidal waveform. To this end, a new - controlled silicon dimmer circuit is proposed. The control of silicon controlled dimmer control is controlled by STM32F407 microcontroller to realize the brightness adjustment of the incandescent lamp. Here using bidirectional thyristor BTA16 as switching device, its characteristic is to have three p-n junction four layer structure of high power semiconductor devices [17], bidirectional thyristor conduction at any point, only on the independent closed. It can be seen that whether or not the zero is accurate is crucial. In order to improve the stability of the thyristor dimmer circuit, every zero point of the ac is needed to ensure that every signal trigger is generated at zero point. In Figure 3, AC_L is the fire line, AC_N is the zero line. The optical coupling TLP521 has the voltage, the internal diode is lit, the transistor conductance, the electricity is completed, the photoelectric conversion process. The fire line has sine wave ac waveform. The following circuit is good to ensure that zero point $\mathrm{A}, \mathrm{B}$ and $\mathrm{C}$ appear when the output signal zero is high. Since the AC_N zero line has always been 0V, the two optoelectronic couplers in FIG. 3 are closed and the internal triode is not conductive, so zero is high level; When A and B are at zero, then the U9's internal diode is lit, and the transistor leads, so zero is low; At point B, the output zero is high as the voltage of B is 0V. However, more than point $\mathrm{B}, \mathrm{AC} \_\mathrm{L}$ input voltage is lower than $0 \mathrm{~V}$, the photoelectric coupler $\mathrm{U} 10$ internal diode is lit, the triode guide, then zero is low level, and all the way to $\mathrm{C}$; At point $\mathrm{C}$, the same thing as A, the output zero is high. The diagram of the relationship between the zero waveform of the circuit and the alternating current is shown in FIG. 4.

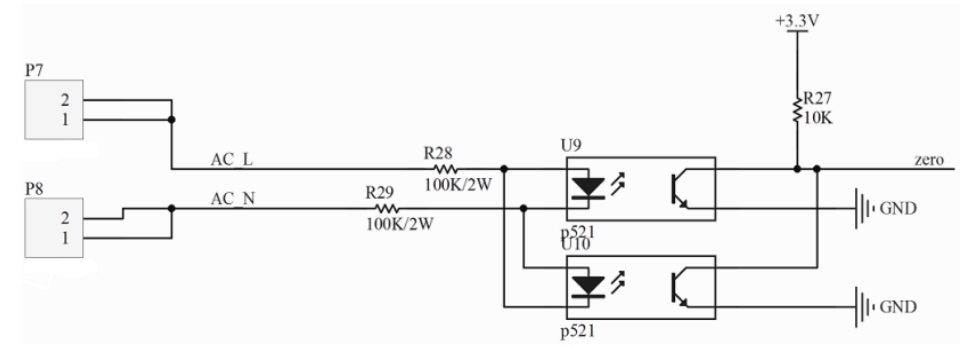

Figure 3. The zero acquisition circuit

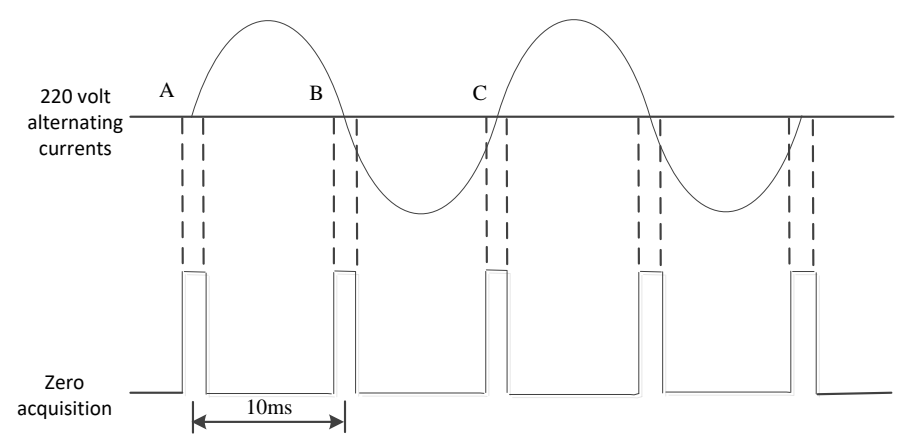

Figure 4. The wave form of zero acquisition

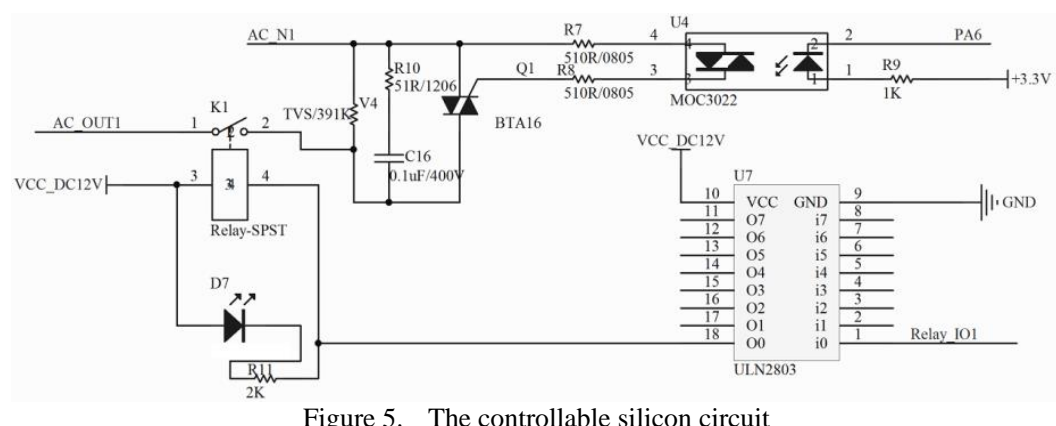

Figure 5. The controllable silicon circuit

Next, the core control section of the triac dimming circuit will be introduced. The photoelectric coupler MOC3022 drives bidirectional BTA16, and the incandescent lamp is connected on the AC-N1 terminal. The whole circuit realizes dimming through controlling the pulse width of STM32F407pin A6 to adjust its duty cycle. In addition, the relay part mainly is the switch control circuit, which was supported by the $12 \mathrm{~V}$ power. When pin RelayIO1 of the U7 inputs high, the corresponding pin 18 outputs low. At this time the relay coil has current, and the switch K1 is closed, and the circuit loop of 
the incandescent lamp is closed, and the indicating lamp D7 is lightened. As the switch control circuit is loop, the PA6 dims. The pin A6 of STM32 controls the on/off of the MOC3022 to realize the turn-on and turn off of the bidirectional BTA16. AC_N1 is alternating current. Since the BTA16 is controlled by two directions, the load incandescent lamp can operate during an AC cycle while the BTA16 is turned on. Among them, the incandescent dimming circuit is shown in Figure 5.

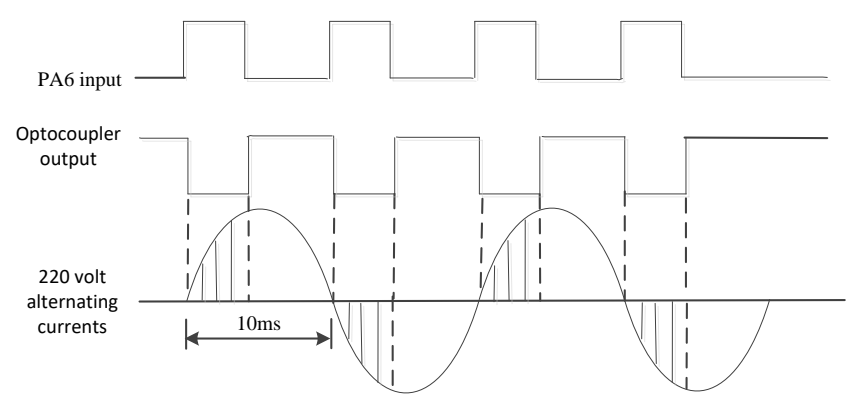

Figure 6. The dimming wave form of controllable silicon

The whole circuit has three waveforms, as shown in Figure 6. Because the PA6 is connected to the negative electrode of the diode inside the optocoupler MOC3022, the output waveform of the optocoupler is opposite to the PA6 input waveform. Since $220 \mathrm{~V}$ alternating current is $50 \mathrm{~Hz}$, only the shaded part in Fig. 6 is enabled to turn on the incandescent lamp. At the same time, when the resolution is more than $50 \mathrm{~Hz}$, the human eye will not recognize, so it can achieve stably dimming [16].

Nowadays, LED also becomes one of the main lamps. As the service company provides the LED built-in drive, adjustable voltage range is $1-10 \mathrm{~V}$, so this will mainly make the LED as a load. 1-10V is an analog dimming mode [13]. In fact, the circuit changes the $1-10 \mathrm{~V}$ voltage signal into PWM dimming signal to achieve the conversion process of analog signal to digital signal, and then control the brightness of LED. The main circuit structure is shown in Figure 7.

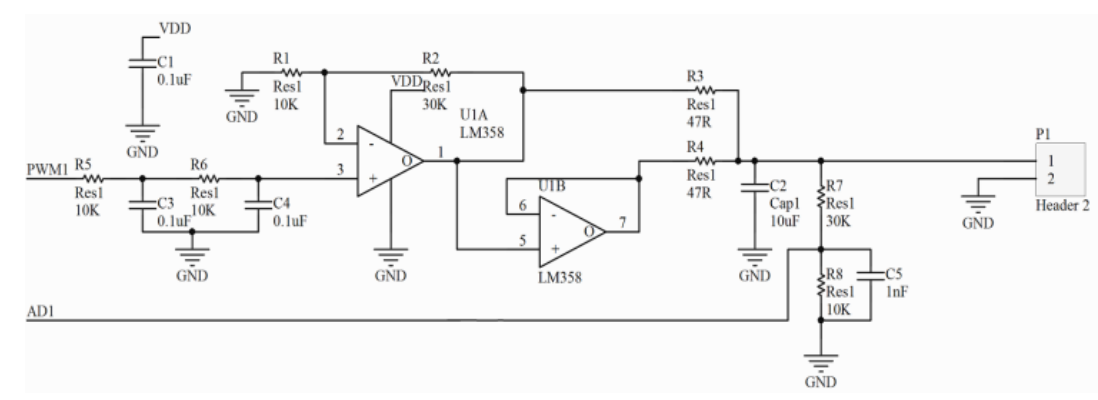

Figure 7. The 1-10V dimming circuit

The dimming circuit is mainly composed of two order RC filter circuit, double operational amplifier LM358, feedback circuit and so on. PWM wave by STM32F407 internal timer signal occupies adjustable signal, after a series of circuit, P1 interface is connected with the load of LED lights, so as to achieve the purpose of regulating light [18]. The whole process is accomplished by converting PWM wave to DA. Since the STM32F407 is powered by 3.3V, the PWM wave with a amplitude of $3.3 \mathrm{~V}$ is generated. This assumes that the duty ratio is $\mathrm{n} \%$, the range of $\mathrm{n}$ is $[0,100]$, after two order filter circuit, the output voltage is $3.3 \mathrm{~V}^{*} \mathrm{n} \%$; The operational amplifier U1A and R1, R2 make voltage amplification effect, the magnification is 4 , then the output voltage amplitude is $3.3 \mathrm{~V} * \mathrm{n} \% * 4$; the operational amplifier U1B is as voltage follower that the output voltage is the same to the input, namely $3.3 \mathrm{~V} * \mathrm{n} \% * 4$. Finally, after R7, R8 and C5, going back to the AD1 (STM32 PA3), the feedback voltage amplitude is $3.3 \mathrm{~V} * \mathrm{n} \% * 4 *(1 / 4)$; P1 interface is connected with LED, while the voltage value is $3.3 \mathrm{~V} * \mathrm{n} \% * 4$. Obviously, it can meet the requirement of $1-10 \mathrm{~V}$ range dimming. The whole circuit has good stability and reliability in practical application.

\subsubsection{Hardware design of acquisition part}

The core of the acquisition terminal is the light intensity sensor acquisition circuit, as shown in Figure 8 . The core of the microcontroller is ARM Cortex-M4. The supporting peripheral circuit includes an external crystal oscillator circuit, a JLINK download interface circuit and a reset circuit and so on [19]. 


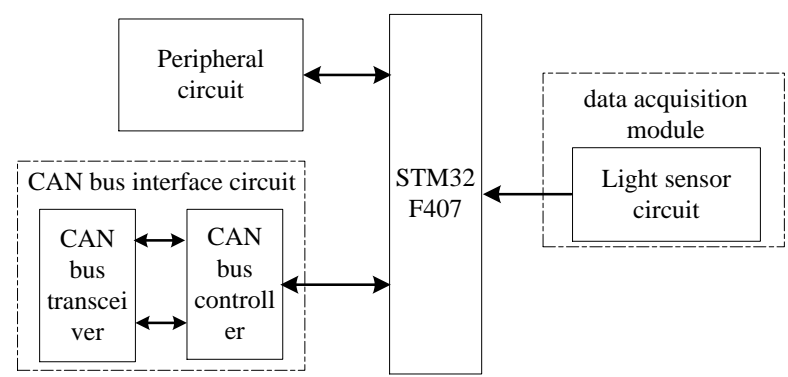

Figure 8. The hardware structure of acquisition part

The core of the acquisition terminal is the light intensity sensor acquisition circuit. A new type of ARM chip based on Coretx-M4 is used as micro controller, and controlling OPT3002 is realized by I2C bus. As the I2C bus interface uses Open Drain (open drain), the three control pins need to pull up the resistance of the $10 \mathrm{~K}$ to pull the signal line to a high level. The LX_SCL is the clock line of the control circuit that provides the OPT3002 clock pulse, and the LX_SDA is the data line that reads data from the sensor. The capacitor in the circuit is mainly used for filtering [9].

\subsection{Software Design of System}

The design of CAN communication software mainly includes the initialization of CAN communication, CAN data receiving and CAN data sending. Initialization is the cornerstone of any branching program. In this module, the receiving and dispatching of CAN data will be the core of the whole communication. First of all, the controller passes the data to be received / sent to the receive / send buffer, then reads the received information through the CAN receive subroutine and executes it. The sending subroutine sends the data that needs to be sent to the bus [20]. A detailed description will be given below.

\subsubsection{CAN Bus Communication Model}

For a common sense network, data communication rules the rules of data exchange between devices in the network. The CAN message is transmitted using the so-called producer consumer model, as shown in Figure 9: device that provides data (e.g., as a multicast or broadcast message) to other devices without being requested [7]. Such communication service is the so-called producer - consumer model.

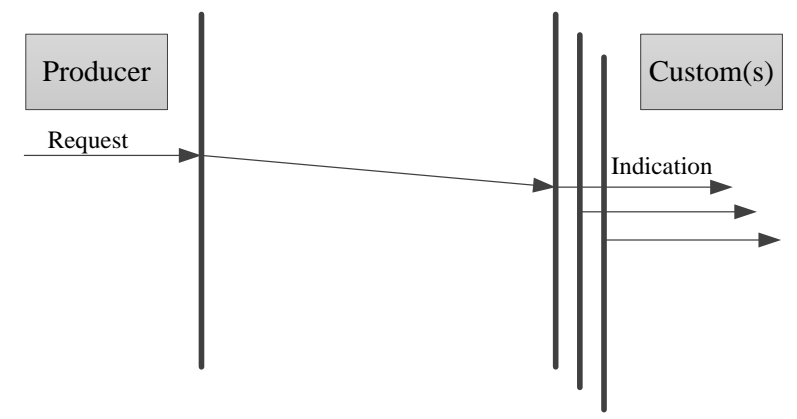

Figure 9. The communication model of CAN-bus

\subsubsection{CAN Data Transmission}

On the premise that the transmit buffer is not locked, the CAN bus sends the data subroutine to judge whether the transmission is successful by querying [8]. And the return value indicates whether the data is to be sent successfully. The sending process is shown in Figure 10. 


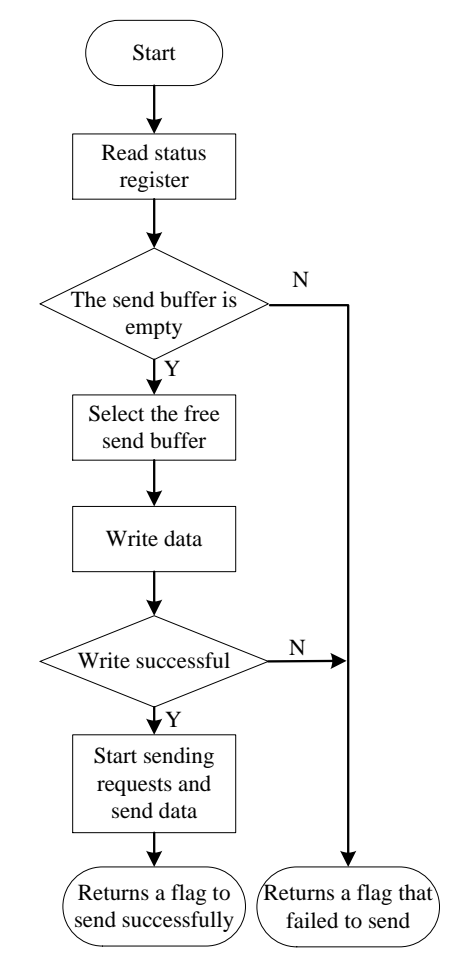

Figure 10. The sending process of CAN

\subsubsection{CAN Data Reception}

CAN bus receives data using interrupt mode, and judges the received data. By executing interrupt handler program, the data is read from the receiving buffer and processed accordingly. The reception process is shown in Figure 11.

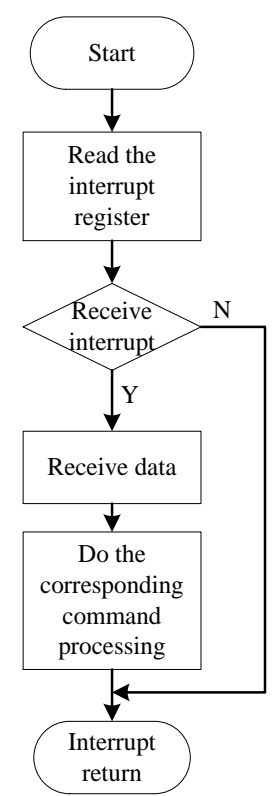

Figure 11. The reception process of CAN

\section{Results}

The relationship between brightness of the incandescent lamp and the duty cycle of pulse width of waveform will be explained here. The main relation is written in MATLAB. The main procedures are as follows. Among them, is duty cycle. The main relationship is shown in Figure 12. 
$\mathrm{t}=[]$

$\mathrm{S}=[]$

for $\mathrm{s}=0: 0.01: 1$

$\mathrm{S}=[\mathrm{S} ; \mathrm{s}]$;

$\mathrm{x}=\operatorname{acos}(1-\mathrm{s})$;

$\mathrm{t}=[\mathrm{t} ; \mathrm{x}]$;

end

figure

$\operatorname{plot}(\mathrm{S}, \mathrm{t})$

xlabel('Duty cycle');

ylabel('Brightness value');

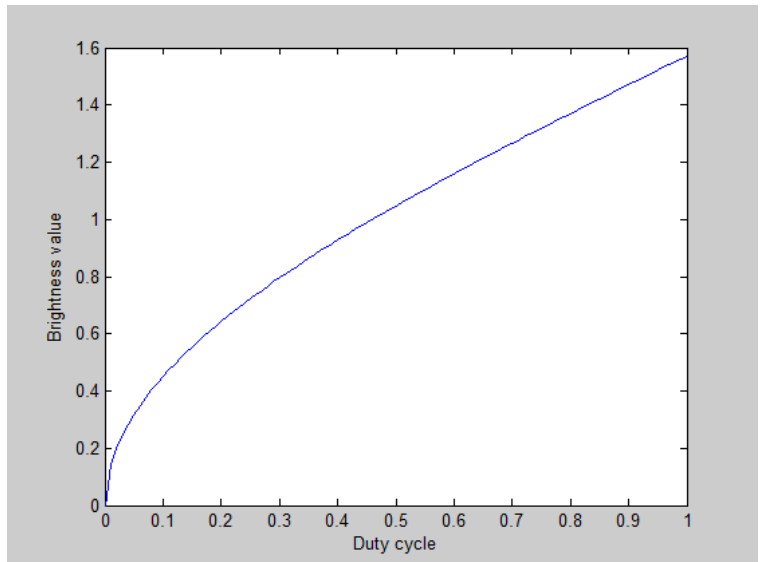

Figure 12. The relationship between luminance and duty cycle of incandescent lamp

The method of integrating sine function is mainly used to adjust the brightness of incandescent lamp. From the realization function, you can know that the integral area is the duty cycle to be adjusted, and then realize the change of brightness. Here, the variable $\mathrm{x}$ is used as the brightness value, and the following relations will be obtained. In Formula 1 , the value range of the variable $y$ is $[0,1]$, which represents the value range of duty cycle that is from 0 to $100 \%$.

$$
\int_{0}^{x} \sin x d x=y
$$

After repeated debugging of software and hardware, the software sets the $10 \mathrm{~ms}$ of pulse cycle, and the accuracy is equal to 1100 , and it uses the normalization method. In this test, the pulse width that is exactly in line with the above theoretical values is obtained. The relation is shown in Table 1. Through the field test data statistics, we can see that under the dimming control of PWM, the output voltage of LED is proportional to the duty cycle of PWM. The relationship between the output voltage and duty cycle is shown in Figure 13. The entire testing process was successful. The results show that the system can collect effectively, transmit and process the data at high speed, and the running results are very good, which fully embodies the advantages of high performance and high reliability.

\begin{tabular}{|c|c|c|c|c|c|c|c|}
\multicolumn{2}{c|}{ Table 1. The relationship between duty cycle and pulse width } \\
\hline Duty cycle & 0 & $10 \%$ & $30 \%$ & $50 \%$ & $70 \%$ & $90 \%$ & $100 \%$ \\
\hline Normalization & 0 & 0.29 & 0.51 & 0.67 & 0.81 & 0.94 & 1 \\
\hline Pulse width & 0 & 316 & 557 & 733 & 887 & 1030 & 1100 \\
\hline Divided by 5.5 & 0 & 57 & 101 & 133 & 161 & 187 & 200 \\
\hline
\end{tabular}




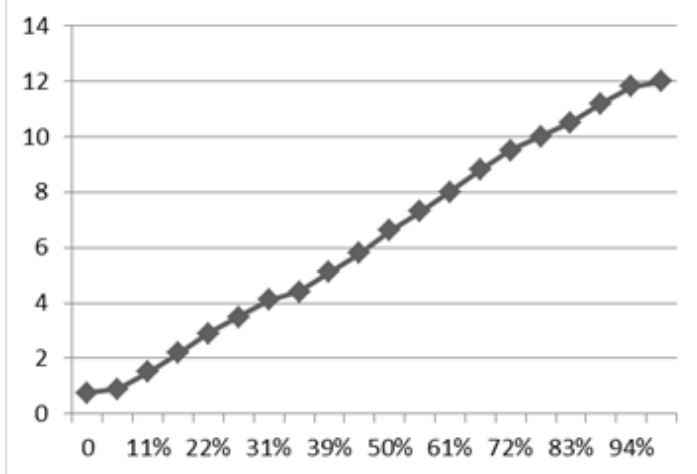

Figure 13. The relation between the output voltage and duty cycle

According to the requirements of the hotel room lighting function, the above experiments show the system function is feasible. The room lighting control system has the following functions.

- Monitoring Function: When the sensor transmits the collected signals to the controller, the controller can process the transmitted signals according to certain rules.

- Automatic Control Function: The system can adjust the brightness of light. When they tested the indoor illuminance reaches the set value can close some lighting equipment or reduce the voltage at the ends of the lighting equipment that lighting levels can reach the most appropriate level, this can not only create a comfortable environment, also can effectively reduce the waste of energy.

- Communication Function: Communication network is an important part of the whole system connection. Each controller is located on each node of the communication network, so as to realize the function of data exchange and command transmission among each node [3].

\section{Conclusions}

This paper presents the design and implementation of room light control system based on ARM Cortex M4 for the first time. The ARM Cortex M4 processor is developed based on the Cortex-M3 kernel, its performance is $20 \%$ higher than Cortex-M3. The system integrates the embedded technology, sensor technology and Internet of things technology to realize the dimming intelligence and accelerates the process of realizing the smart room. After a multitude of field tests, the design of the data acquisition and control system is successful. The design solves the energy wasting problem fundamentally. For example, the traditional light intensity is not enough; people always turn on the light in the dark and forget to turn it off later [4]. The whole system can realize management and maintenance of the lighting system simply, and be more flexible in adapting to the future lighting layout and changing of control mode [5]. Smart rooms lighting control system has a benchmark role in the construction industry.

\section{Acknowledgements}

This work was supported by the development of the smart hotel modular guest room control system, which belongs to the Prospective Joint Research Project of Jiangsu Province under Grant BY2016053-07, Innovative training program for college students in Jiangsu (201710304051Z), Nantong science and technology project (CP22014005) and Nantong science and technology project (AA2014013). The authors thank the 3 anonymous reviewers for their helpful suggestions.

\section{References}

1. Apostol, Mihaela Simona. "Hotel Lighting Systems in the Context of Global Energy Crisis - An Approach in Perspective of Sustainable Development." International Journal of Modern Physics E, vol.20, no.12, pp.84-84, 2012.

2. Y Guo, Q Wu and H Wang, "Design and Implementation of Intelligent Headlamps Control System Based on CAN bus" International Conference on Systems and Informatics. IEEE, pp. 385-389, 2012.

3. $\mathrm{Na} \mathrm{Gu}$, "Research and Design of Intelligent Lighting Control System Based on CAN bus." Jiangsu University, 2007.

4. F Guo and J Y Duan, "Design and Implementation of Intelligent Baby Room Monitoring System Based on the Internet of Things.” Applied Mechanics \& Materials, vols. 513-517, pp. 2484-2486, 2014.

5. Kasahara, Yoshihiro, M. Miki, and M. Yoshimi. "Preliminary Evaluation of the Intelligent Lighting System with Distributed 
Control Modules." International Conference on Intelligent Systems Design and Applications IEEE, pp.283-288, 2012.

6. M Li and H J Lin, "Design and Implementation of Smart Home Control Systems Based on Wireless Sensor Networks and Power Line Communications." IEEE Transactions on Industrial Electronics, vol. 62, no. 7, pp. 4430-4442, 2015.

7. Y Liang ,etc. "A New TRIAC Dimmable LED Driver Control Method Achieves High-PF and Quality-of-Light." Applied Power Electronics Conference, 2012.

8. K. H Loo, et al. "On the Color Stability of Phosphor-Converted White LEDs under DC, PWM, and Bilevel Drive." IEEE Transactions on Power Electronics, vol.27, no.2, pp.974-984, 2012.

9. M Miki, et al. "Construction of Illuminance Distribution Measurement System and Evaluation of Illuminance Convergence in Intelligent Lighting System.” vol.9, no.1, pp.2431-2434, 2010.

10. Qu, Guoqing, F. Xiong, and J. Li. "Applications and Platform of Technology-driven Financial Innovation and Integration Patterns for LED Industrial Chain of Integrated Business." 2015 information technology and mechatronics engineering conference, 2015.

11. Siddiqui, Adeel A., et al. "ZigBee Based Energy Efficient outdoor Lighting Control System." International Conference on Advanced Communication Technology IEEE, 2012.

12. G Strazdins, Elsts A, Nesenbergs K and et al, "Wireless Sensor Network Operating System Design Rules Based on Real-World Deployment Survey.” Journal of Sensor \& Actuator Networks, vol. 2, no.3, pp. 509-556, 2013.

13. H Xu and Kim C W, "Design and Implementation of LED Lighting Control System Using Arduino Yun and Cloud in IoT." vol. 11, no. 10, pp. 983-988, 2016.

14. T Zhang and K B Jia, "Design and Implementation of IDC Engine Room Intelligent Management and Control System Based on Data Mining” International Conference on Computer Science and Service System, IEEE Computer Society, pp. 1366-1369, 2012.

15. Junji Zhang, and Yanxia Shen. "New LED Dimming System Based on the MSP430."Journal of Jiangnan University, 2014.

16. Q Zheng. "The design and the Implementation of Communication Gateway between CAN Bus and Ethernet." Advanced Information Technology, Electronic and Automation Control Conference IEEE, pp.862-866, 2016.

17. J. Zhang, et al. "Primary side Feedforward Control for TRIAC Dimmable Light Emitting Diode Driver with Constant Power." Iet Power Electronics, vol.6, no.3, pp.572-580, 2013.

18. Zhang, Ruihong, and S. H. Chung. "A TRIAC-Dimmable LED Lamp Driver with Wide Dimming Range." IEEE Transactions on Power Electronics, vol. 29, no.3, pp.1434-1446, 2013.

19. J Zhang, H. Zeng, and T. Jiang. "A Primary-Side Control Scheme for High-Power-Factor LED Driver with TRIAC Dimming Capability." IEEE Transactions on Power Electronics, vol.27, no.11, pp.4619-4629, 2012.

20. Q Zhang, Q Qiu and X Chen, "Design and Implementation of A Smart Networking Control System for LED Lighting Based on CAN.” International Journal of Wireless \& Mobile Computing, vol. 6, no. 6, pp. 529-534, 2013.

Jiajia Feng is now studying in the school of electronic information at Nantong University. She is a graduate student. His main research interests include Internet of things and embedded systems.

Yongjie Yang is a graduate of Nanjing University of Science and Technology, holds the post of dean of the school of electronic information, Nantong University. He has participated in the national and provincial studies. The main research direction is wireless communication

Hongming Shen graduated from Xuzhou Normal University. He is a Ph. D. student in the school of electronic information, Nantong University. He is studying in the United States.

Zhitian Cai is an undergraduate student at the school of electronic and information engineering at Nantong University, has participated in academic competitions. 